\title{
Multi Scale Hashing Access in Multi-Dimensional Datasets using Bootstrapping
}

\author{
S.Jayaprada, J.Ranga Rao, M.Srilatha
}

\begin{abstract}
The query is the most widely used in many applications. Computation of multi-dimensional dataset is more complication by using existing algorithms. Showing the spatial results by using the multi-dimensional dataset is implemented in this paper. The existing system ProMiSH (Projection and Multi Scale Hashing) is not performed based on the keyword query searching. The proposed system Ensemble approach with bootstrapping algorithm shows better results. The implementation is done with a spatial dataset with a location-based query keyword search done in the proposed system.
\end{abstract}

Keywords: NKS Querying, multi-dimensional data, indexing, ProMiSH, Bootstrapping.

\section{INTRODUCTION}

Nearest Keyword set studies on content-rich dissimilar types of data sets. The NKS review is an procedure of catchphrases in outlook of the theme. Also, the preparation of the query unites ' $\mathrm{K}$ " type of catchphrases as a cluster and focusses each and each set which groups data based lots along with assemblies in which bunches of the multi-dimensional region is shaped. Each point is considered with an preparation of collections.

When all is said in finished, ProMiSHA is more time and space operative associated to ProMiSHE which can get close model consequences practically communication. The file structure and the hunting method for ProMiSHA are like ProMiSHE, beside these lines, we just represent the differences in the events. Here the design of ProMiSHA varies with ProMiSHE by the process for allocating projection section of irregular bits of path space. ProMiSH and parts projection area into containers of alike width which are not enclosed, not at all similar ProMiSHE bundling projections of words into casing repositories.

Therefore, every evidence sets get one repository point from an unequal vector $\mathrm{z}$ in ProMiSHA procedure. Only a private check is created due to an reminder of own section focuses created by every single m optional vectors. Every solitary id is complete using its cast in the path space.

\section{LITERATURE SURVEY}

Zhisheng et. al [1], anticipated a geographic inquiry that is completed out of question watchwords then an area, a geographical hunt engine recuperates archives that are the principal literarily and spatially apropos to the inquiry catchphrases and in this manner consider the area, autonomously, and positions the recouped records as showed by their combined issue and three-dimensional position's to the inquiry. The deficiency of an actual record which will all the though switch each the issue and three-dimensional segments of the reports makes existing geographic pursuit engines inefficient in noticing physical request amid. This paper, we have a tendency to propose a proficient record, alluded to as IR-tree, that adjacent to a best $\mathrm{k}$ report look algorithmic program supports four essential undertakings in archive seeks, to be particular, 1) spatial separating, 2) matter sifting, 3) association calculation, and 4) record positioning in a completely planned approach, and what is a ton of, IR-tree grants inquiries to grasp various masses on issue and three-dimensional association of records at the runtime and amid. This approach prepares for an extensive combination of usages and a procedure of careful inspections done a concentrated sort of things takes coordinated and in this manner, the provisional derives concerning demonstrate that IR-tree strokes the main advantage methods for physical archive seeks.

Christian et al. [2] arranged the area mindful watchword question return stratified items that are almost \{a question|a question |a question\} position which has composed depictions that match inquiry catchphrases. This inquiry happens coherently in a few assortments of malleable and traditionalist net establishments and claims, e.g. telephone directory and Plots organizations. Past effort reflects the possible outcomes of such an inquiry as existence self-governing when positioning. Regardless, a appropriate conclusion question with neighbouring items that are correspondingly pertinent to the inquiry is presumably successful to stay perfect ended an essential dissent while not vital near to matters.

V. Singh et al. [3] arranged standard net is securing a geospatial measurement, intelligences are being spatial, and spatial archived dissents, for instance, elements of interest are associated with partaking content archives then

Revised Version Manuscript Received on 10 September, 2019.

S.Jayaprada, Department of Computer Science \& Engineering, Gudlavalleru Engineering College (Autonomous), Gudlavalleru, Andhra Pradesh, India. (email: jayaprada79@yahoo.com)

J.Ranga Rao, Department of Computer Science \& Engineering, V.R Siddhartha Engineering College (Autonomous), Vijayawada, Andhra Pradesh, India. (email: rangaraoj@gmail.com)

M.Srilatha, Department of Computer Science \& Engineering, V.R. Siddhartha Engineering College (Autonomous), Vijayawada, Andhra Pradesh, India. (email: srilatha.manam@gmail.com). 
accompanying blend of geo-area and intelligences enables additional sensibly top_k question that takes into greatest every area closeness and substance essentialness. To data, generally, resident frameworks occur that worth enlisting a over-all net information recuperation question though also bringing an area into the record. This paper proposes another requesting structure for area cognizant best $\mathrm{k}$ content recuperation. The system impacts the agitated record for contented recuperation and accordingly the $\mathrm{R}$-tree for three-dimensional vicinity questioning.

Chakrabarti et al. [4] referred the customers customarily look three-dimensional files alike yellow page material by catchwords to and associations adjacent to their stream area. Such inquiries stand more being done from PDAs. Comprising the whole inquiry is extensive then slanted to botches, quite from mobile phones, and tend to report this issue by showing kind ahead pursuit quality on three-dimensional databases. Like watchword investigate on three-dimensional information, type-ahead search should be extent aware, i.e, with each note being typewritten, it must backpedal three-dimensional things whose terms (or depictions) are strong culmination of the inquiry sequence typewritten amid this approach, and that abundant most hoisted as path as closeness to customer's area and elective inert scores. Current answers for type gaining inquiry can't be employed particularly as they're not area vigilant. We have a tendency to display that a straight forward blend of present frameworks for playing compose fast scan with those for playing separation look achieve insufficiently.

Zhang et al. [5] arranged Mapping mixture are rising net two.0 submissions amid which data matters, for example, destinations, pictures, and chronicles from shifted sources are consolidated and set apart in an exceptionally direct using arthropod sort that are released by net-based for the most part mapping courses of action, for example, Google and Yahoo Maps. These articles are generally associated with a preparation of names getting the put in phonetics and a preparation of directions demonstrating topographical areas.

Z. Li et al. [6] blend closest catchphrase look in spatial records, in Asia-Pacific net Conference, 2010. Watchword look on relative databases is valuable and in vogue aimed at a few clients while not specialized foundation. As of late, blend watchword look on relative databases was arranged and has pulled in intrigue. Be that as it may, 2 essential issues still remain. To begin with, blend watchword inquiry will be horribly expensive on enormous relative databases, part since of the absence of efficient files. Second, the best k Answers to a blend catchphrase question has not been self-tended to reliably, together with each the positioning model and consequently the practical investigation techniques.

DE Felipe et al. [7] a few requests need discovering matters nearby to a particular area that contains a gathering of catchphrases. for instance, connected phone directory allows customers to control AN report and a gathering of catchphrases. Consequently, the client gets a posting of groups whose depiction covers these watchwords, entreated by their parting from the coveted discourse. The issues of closest neighbour look at spatial information and catchphrase seek on content learning are widely considered on an individual premise. Notwithstanding, to the least complex of our data there's no sparing technique to answer spatial

watchword inquiries, that is, questions that determine each an area and accumulation of catchphrases.

M. Datar et al. [8] given a one of a kind Locality-Sensitive Confusing topic for the Estimated Nearest Neighbour drawback underneath record standard, bolstered stable dispersions. Our subject enhances the day and age of the sooner algorithmic program for the instance of the 12 standard. It moreover yields the essential recognized obviously sparing surmised NN algorithmic program for the case $\mathrm{p}<$ one. we have a tendency to furthermore demonstrate that the algorithmic program discoveries the precise close neighbor in $\mathrm{O}(\log \mathrm{n})$ time for information fulfilling convinced restricted growth\| state rather than prior plans, our LSH topic works straightforwardly on focuses inside the metric space while not embeddings.

M. Kleinberg et al. [9] Develop new way to deal with closest neighbor drawback, bolstered a route for joining aimlessly picked one dimensional plans of the hidden reason set. Dual algorithmic projects are present amid this underlying for result alphabetic character rough closest neighbors and second alphabetic character inexact closest neighbor calculation with close straight stockpiling and question time enhances asymptotically direct pursuit by and large measurements.

Aristides Gionis et al. [10] look at an extraordinary subject for rough comparability seek upheld hashing. The fundamental arrangement is to muddle the focuses from the data thusly to confirm that the possibility of a crash is developed for matters that are almost each other than for the individuals who are far separated. Strategy stretches imperative change in day and age over elective methodologies aimed at looking in immense dimensional territories bolstered stratified tree de-creation. This topic scales well notwithstanding for nearly sizable measure of extents (more than 50). Past system [6] illuminate this drawback speedily only for the estimated event right and temperate near neighbor Search in High Dimensional zones. This is styles to determine r-close neighbor inquiries for a set inquiry change or for a set of question choices by probabilistic certifications and afterward reach out for closest relevant questions.

\section{METHODOLOGY \& RESULTS}

Utilizing the mix of R-tree and transformed list, the area particular catchphrase inquiries were replied on network and GIS framework. Positioning to items were finished in current framework i.e. IR2 tree to rank items in light of the mix of their partings to the inquiry area and the importance of contented portrayal to the question watchwords. Utilizations catchphrase thickness and correct coordinating for watchword parsing procedure. We can look maybe a couple of pictures identified with the watchword not extra than that. Cao et al., and long et al., anticipated calculation to recover a gathering of three-dimensional web protests with the end goal that the gathering catchphrases cover the inquiry watchwords and the articles have most minimal between question separations. Other related inquiries incorporate total 
closest watchwords seek in three-dimensional databases, top-k particular question, top-k destinations in a spatial information base, an ideal area questions.

\subsection{Novel Multi Scale Hashing Approach Algorithm}

Novel Multi Scale Hashing Approach Algorithm utilizes the syntactic data set is composed from various data resources. In which we gather location and item examine with distance calculation between the source and destinations by using a boot strapping algorithm, with an established keywords that are resultant from tags. The question co-ordinates play an essential role in each phase of the algorithm to trim investigation space. Our effort deals with if keyword as an input. In the proposed approach which is a new multi-scale catalogue for precise and near NKS enquiry dispensation and progress effective search procedures that effort with the multi-scale directories for fast query processing. Distance looking is also very informal with R-trees. In detail, the best-first process is accurately considered to yield data ideas in rising directive of their reserves. In order to run the submission resourcefully, the user must have subsequent features. User offers the keyword i,e items as an input.

\subsection{Algorithm:}

The proposed bootstrapping algorithm has the resulting steps:

1. For the state of interest, recognize related results and calculate the equivalent values for every instance in the three-dimensional sample, $\mathrm{W}$. Let $\mathrm{Xj}$ be the path of values for the connected findings of places with items $\mathrm{j}$.

2. Set $\mathrm{Dj}$ to the number of encounters with connected ICD-9 codes for substances $\mathrm{j}$

3. Recognize a training set $\mathrm{T}$ since $\mathrm{W}$, covering positive and negative instances.

4. Set $\mathrm{Cj}$ (the period of items $\mathrm{j}$ ) to $\mathrm{Dj}$

5. Initiate iterative procedure (superscript signifies iteration quantity):

(a) Using $\mathrm{T}$, form support vector machine built classification prototypes to acquire non-linear relationship $\mathrm{C}^{\wedge} \mathrm{ji}=\mathrm{fi}(\mathrm{Xj})$, where $\mathrm{C}^{\wedge} \mathrm{ji}$ is the prototype's estimate for $\mathrm{Cj}$

(b) Relate fi $(\mathrm{Xj})$ to $\mathrm{W}$ and produce histogram over $\mathrm{C}^{\wedge} \mathrm{ji}$ for all three-dimensional items in $\mathrm{W}$;

(c) Choice a limit threshold $\eta$ i to separate the constructive and negative populaces.

(d) Set $\mathrm{Lji}=1$ if $\mathrm{C}^{\wedge} \mathrm{ji}>\eta \mathrm{i}$ and $\mathrm{Lji}=0$ then, here, $\mathrm{Lji}$ is our estimation of the patient's marker and a positive marker specifies the patient is constructive for the state. Message that at initialization, $\mathrm{Lji}=1$ if $\mathrm{Cj}>0$; else $\mathrm{Lj} 0=0$.

(e) Relate Lji to Lji-1 and calculate Fi, the proportion of patients for whom the marker has different in the present iteration.

(f) If $\mathrm{Fi}<\delta$ (where $\delta$ is an acceptable change threshold), return Lji and terminate; else, update $\mathrm{Cji}+1=\left[\mathrm{i} * \mathrm{C}^{\wedge} \mathrm{ji}+\mathrm{D}\right] /(\mathrm{i}+1)$ for all $\mathrm{j}$ in $\mathrm{T}$

We start with the index for exact search. There are 2 main component included i.e. Inverted index ikp and Hash table inverted index pairs (HI). We treat keyword as keys and provide it as an input to our system. There are hash bucket
IDs and respective points associated with the keywords, it will find all the hash buckets in IKHB (keyword bucket inverted index), having all query keywords.

In our system we are performing subset search on each retrieved hash bucket using points having query keywords. These indexes fail to scale dimension greater than 10 because of its dimensionality thus random projection with hashing and indexing has come up in the method of nearest keyword search in multidimensional datasets.

For e.g. Contemplate there are 3 keywords a, b, c. then pointed the opinions connected with the hash container IDS i.e. near will be examination for all the keywords, if there is no precise match for the keyword, then it will examine for two keywords i.e. we became the numerous grouping of the keywords, and before for the only keyword. Therefore all the keywords are examined proficiently with less period and more accurateness in multidimensional datasets, and we suggested solution re-implementing numerous sequences in the top $\mathrm{k}$ adjacent set in multidimensional datasets.

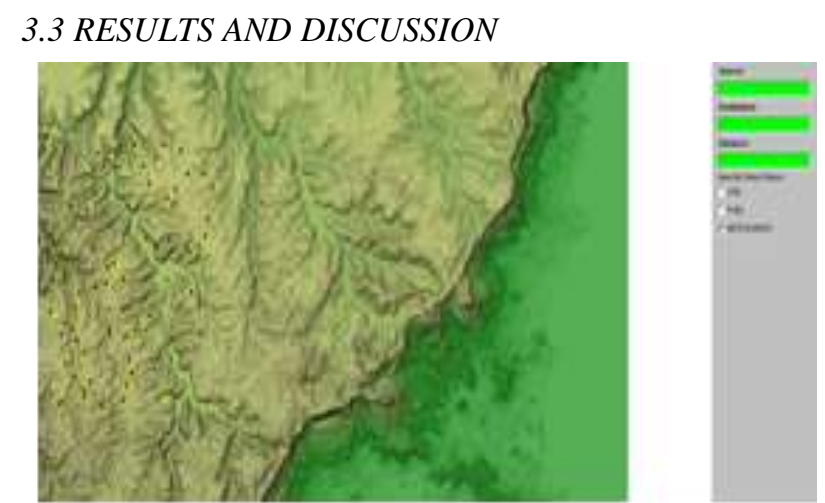

Figure 1 Spatial Dataset display

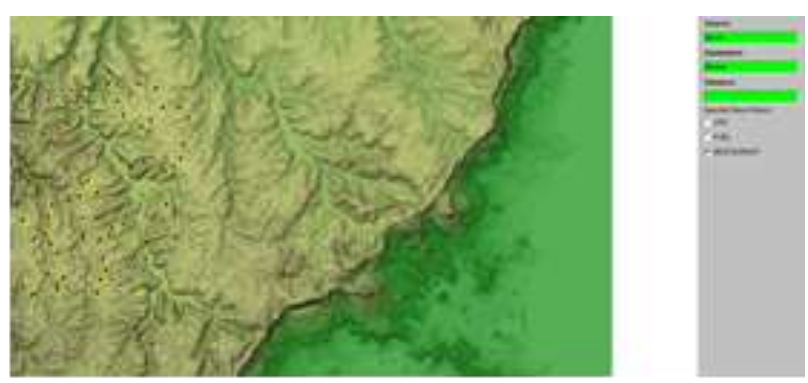

Figure 2 loading Spatial Data with number of Restaurants

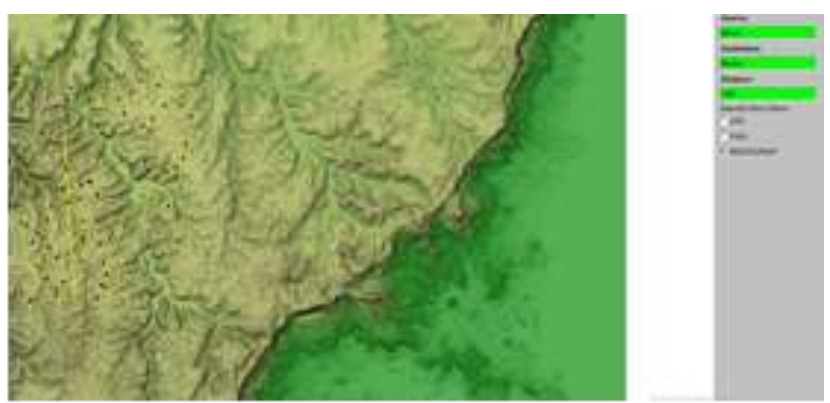

Figure 3 Routing between source and destination Blue Eyes Intelligence Engineering 
Simulating Events in Proposed System

Acquiring information about all places

Perry@ (314.0,29.0)

Glenwood@(259.0,169.0)

Hartwell@(67.0,430.0)

Unionville@(328.0,455.0)

Lagrange @ (321.0,263.0)

Kinderlou @ (200.0,22.0)

Sycamore @ (115.0,29.0)

Tarver@ (99.0,342.0)

Valdosta@(75.0,87.0)

Rebecca@ (170.0,146.0)

Baxley@ (180.0,260.0)

Euharlee @ (65.0,276.0)

Denmark@(219.0,71.0)

Millen@(228.0,489.0)

Tyrone@ (366.0,289.0)

Tucker@(66.0,136.0)

Everitt@ (137.0,423.0)

Dalton@ (216.0,46.0)

Edith@ (213.0,93.0)

Kite@ (291.0,464.0)

Saint@ (45.0,230.0)

Rutledge @ (176.0,445.0)

Cecil@ (139.0,381.0)

Gainesville @ (88.0,145.0)

Howell @ (342.0,414.0)

Pembroke@ (88.0,264.0)

Commerce@ (53.0,171.0)

Pelham@ (10.0,99.0)

Marietta@ (35.0,494.0)

Dudley@(212.0,358.0)

Portal @ (129.0,54.0)

Villa@(381.0,307.0)

Cox@(272.0,285.0)

Cedartown@(163.0,226.0)

Westwood@ (40.0,175.0)

Enigma@ (145.0,450.0)

Centerville@(316.0,388.0)

Thomaston@(200.0,233.0)

Mcrae@(100.0,126.0)

Buchanan @ (96.0,182.0)

Atlanta@ (273.0,442.0)

Chula@ (228.0,146.0)

Buena@ (346.0,279.0)

Coverdale @ (224.0,466.0)

Manassas@ (305.0,124.0)

Nankin@ (368.0,191.0)

Montezuma@(252.0,305.0)

Snellville@(62.0,272.0)

Surrency@ (170.0,137.0)

Oxford@(209.0,49.0)

Dixie @ (257.0,398.0)

Baden@(130.0,361.0)

Broxton@ (371.0,137.0)

Walthourville@ (308.0,129.0)

Ellijay@(188.0,258.0)

Thunderbolt @ (377.0,416.0)

Hazlehurst @ (116.0,106.0)

Moultrie @ (395.0,393.0)

Janis@ (169.0,180.0)
Byron@ (154.0,12.0)

Rome@ (32.0,490.0)

Preston@(293.0,23.0)

Ochlocknee@ (141.0,218.0)

Lincolnton@ (301.0,300.0)

Osterfield @ (364.0,272.0)

Blue@ (114.0,40.0)

Nelson@ (168.0,253.0)

Abba@ (309.0,280.0)

Odum@(43.0,32.0)

Amboy @ (94.0,62.0)

Byromville @ (386.0,336.0)

Donalsonville@(312.0,363.0)

Cogdell@(200.0,179.0)

Stilson@(244.0,428.0)

Braselton@ (349.0,209.0)

Iron@(296.0,373.0)

Ousley @ (32.0,332.0)

Alapaha@ (67.0,21.0)

Louisville@ (268.0,77.0)

Ailey@ $(60.0,15.0)$

Lenox@(86.0,358.0)

Bronwood@ (105.0,208.0)

Montrose @ (206.0,133.0)

Culloden@(232.0,157.0)

Austell @ (75.0,156.0)

Dunwoody@ (183.0,427.0)

Statenville@ (211.0,183.0)

Brunswick @ (135.0,287.0)

Ocilla@ (263.0,184.0)

Optimal Location Identified : Denmark

Optimal Location Identified : Ocilla

Optimal Location Identified : Baden

Optimal Distance Identified Between Source(Byron) and

Destination(Baden) is :199

Spatial Text Queries Completed...

***************************************************

$* * * * * * * * * * * * * *$

Starting Arbitrary Constraints Identification and Filtration Process...

Found RESTAURANT As Requested

Details

**********************************

Name: Baden at a distance: 199.0 from your location $* * * * * * * * * * * * * * * * * * * * * * * * * * * * *$

Total Time: 7.660160951 seconds

\section{CONCLUSIONS}

In this paper, the proposed system provides accurate results in multiple keyword searches. This is how consumer data can be used to improve search slope and to find interest of the employer. The proposed system has societal comments will be valuable in the arena of complex word search, and produce the optimization as regular huge size of data obtainable for thorough by attention will be the future for exploration engines. The key benefit of this classification will save lacks of processor cycles used in multidimensional data sets for

Published By: 
finding better keyword search and find the routing between source and destination.

\section{REFERENCES}

1. Zhicheng $\mathrm{Li}, \mathrm{Hu} \mathrm{Xu}$, Yansheng $\mathrm{Lu}$, Ailing Qian,"Aggregate Nearest Keyword Search in Spatial Databases," IEEE 12th International Asia-Pacific Web Conference, 2010.

2. Cao, Xin, Gao Cong, and Christian S. Jensen. "Retrieving top-k prestige-based relevant spatial web objects." Proceedings of the VLDB Endowment 3, pp: 373-384, 2010.

3. V. Singh, S. Venkatesha, and A. K. Singh, "Geo-clustering of images with missing geo tags," in Proc. IEEE Int. Conf. Granular Compute, pp. 420-425, 2010.

4. Basu Roy, Senjuti, and Kaushik Chakrabarti. "Location-aware type ahead search on spatial databases: semantics and efficiency." In Proceedings of the 2011 ACM SIGMOD International Conference on Management of data, pp. 361-372. ACM, 2011.

5. Zhang, Dongxiang, Beng Chin Ooi, and Anthony $\mathrm{KH}$ Tung. "Locating mapped resources in web 2.0." In Data Engineering (ICDE), 2010 IEEE 26th International Conference on, pp. 521-532.

6. Li, Zhisheng, Ken CK Lee, Baihua Zheng, Wang-Chien Lee, Dik Lee, and Xufa Wang. "Ir-tree: An efficient index for geographic document search." IEEE Transactions on Knowledge and Data Engineering 23, no. 4: 585-599, 2011.

7. D. Felipe, V. Hristidis, and N. Rishe, "Keyword search on spatial databases," IEEE 24th Int. Conf. Data Eng.,pp. 656- 665, 2008

8. M. Datar, N. Immorlica, P. Indyk, and V. S. Mirrokni, "Locality-sensitive hashing scheme based on p-stable distributions," in SCG, 2004.

9. KLEINBERG, J. M. 1997. Two algorithms for nearest-neighbor search in high dimensions. In STOC '97: Proceedings of the twenty-ninth annual ACM symposium on Theory of computing ,New York, NY, USA, pp. 599608. ACM Press, 1997.

10. Aristides Gionis, Piotr Indyk, Rajeev Motwani,"Similarity Search in High Dimensions via Hashing",pp: 518-529,VLDB 1999.

11. W. Li and C. X. Chen, "Efficient data modeling and querying system for multi-dimensional spatial data", in GIS, pp. 58:1-58:4,2008.

12. M. L. Yiu, X. Dai, N. Mamoulis, and M. Vaitis, "Best k spatial inclination inquiries", in Proc. IEEE 23rd Int. Conf. Information Eng., pp. 1076- 1085, 2007.

13. Ashlesh S. Patole; Shripadrao Biradar " A Survey on Best Keyword Cover Search “ IJIRCCE Vol. 3, Issue 11, SSN(Online): 2320-9801 ISSN (Print): 2320-9798,2015.

14. Ke Deng; Xin Li; Jiaheng Lu; Xiaofang Zhou," Best Keyword Cover Search" Knowledge and Data Engineering, IEEE Transactions on Year: 2015.

15. Cong, Gao, Christian S. Jensen, and Dingming Wu. "Efficient retrieval of the top-k most relevant spatial web objects." Proceedings of the VLDB Endowment 2, no. 1 (2009): 337-348. IEEE, 2010. 